\title{
Spatial Control of Processing Plasmas in a Multicusp Plasma Source Equipped with a Movable Magnetic Filter
}

\author{
Member Osamu Fukumasa (Yamaguchi University) \\ Member Hiroshi Naitou (Yamaguchi University) \\ Member Satoshi Sakiyama (Yamaguchi University)
}

\begin{abstract}
It is confirmed, with the use of both a movable magnetic filter and a plasma grid, that plasma parameters $\left(\mathrm{H}_{2}-\mathrm{CH}_{4}\right.$ or $\mathrm{Ar}-\mathrm{CH}_{4}$ plasmas) are spatially well controlled. At any filter position, plasma parameters change steeply across the magnetic filter. Then, a plasma source is divided into two parts, i. e. a source plasma region (high density plasma with energetic electrons) and a diffused plasma region (low density and low temperature plasma without energetic electrons). The former is suitable for producing the reactive species and the latter for preparing thin films. In addition, plasma parameters in the diffused plasma are controlled by changing the plasma grid potential. The role of the magnetic filter (i. e. preferential reflection of energetic electrons) is also discussed by computer simulation.
\end{abstract}

Key words : Plasma processing, Multicusp plasma source, Magnetic filter, Electron energy distribution function, Spatial control

\section{Introduction}

The plasma chemical vapor deposition ( $p$-CVD) method has been successfully used in the preparation of various kinds of thin film, e. g. hydrogenated amorphous silicon films and hydrogenated amorphous carbon films. Since the initial processes, the decomposition of source gas molecules or production of reactive species, are triggered by electron collisions, plasma parameters, especially energy distribution and density of electrons, must initially be carefully controlled ${ }^{(1)}$.

So far, we have studied application feasibility of a magnetically filtered multicusp plasma source to a p-CVD reactor. The multicusp plasma source has demonstrated its ability to produce large volumes of uniform and quiescent plasmas ${ }^{(2)}$. Recently, we have shown that the addition of a movable magnetic filter ${ }^{(3)(4)}$ and a plasma grid $^{(5)}$ can spatially control well plasma parameters including electron energy distribution. In this paper, we study further the control of reactive plasmas (i. e. $\mathrm{H}_{2}-\mathrm{CH}_{4}$ and $\mathrm{Ar}$ $\mathrm{CH}_{4}$ plasmas) and the role of the magnetic filter ${ }^{(6)}$.

\section{Experimental apparatus and procedure}

Fig. 1 shows a schematic drawing of the apparatus. The source chamber $(16 \mathrm{~cm}$ diameter, $30 \mathrm{~cm}$ long) is made of stainless steel and is surrounded externally by 16 columns of ferrite permanent magnets which form a longitudinal linecusp configuration for primary electrons and produced plasmas confinements.

Hydrogen or argon gas was introduced into the source chamber continuously. The source pressure $p$ was measured by an ionization gauge. A steadystate hydrogen or argon plasma was produced by primary electrons emitted from 4 tungsten filaments $(0.27 \mathrm{~mm}$ diameter, $10 \mathrm{~cm}$ long $)$. These filaments were biased negatively to a discharge voltage $V_{d}$ with respect to the grounded chamber wall. The 


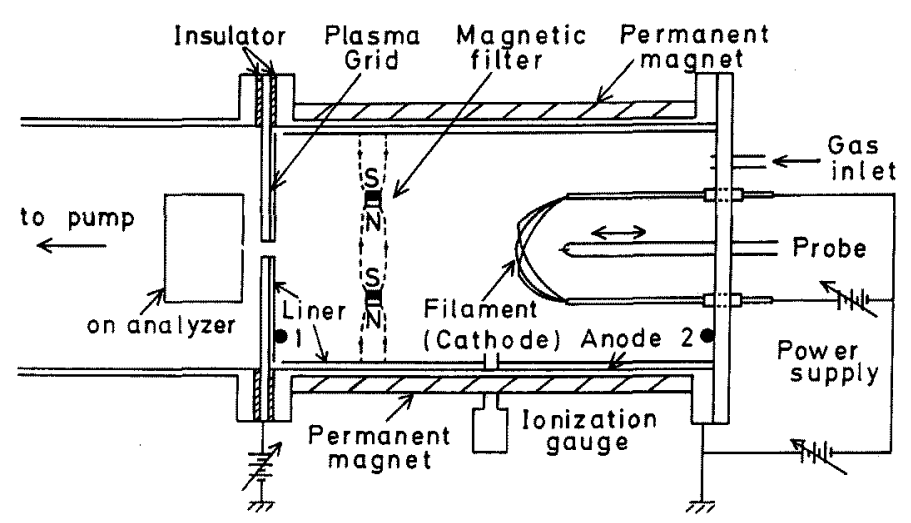

Fig. 1. Schematic diagram of the multicusp plasma source equipped with the movable magnetic filter and the plasma grid whose potential is variable.

entire chamber wall, together with the metal liner, served as the anode for the discharge.

Plasma parameters were measured using two cylindrical Langmuir probes $(0.5 \mathrm{~mm}$ diameter, $2 \mathrm{~mm}$ long). To obtain an electron energy distribution function (EEDF) using the Druyvesteyn method, the second derivative of the probe characteristic was also measured.

A samarium-cobalt magnetic filter ${ }^{(2)}$ divides the entire chamber into an arc discharge (the righthand side) and a diffused plasma (the left-hand side). The filter consists of two rods separated about $55 \mathrm{~mm}$. The maximum intensity of the magnetic field at the mid-plane is about $50 \mathrm{G}$. This filter provides a limited region of transverse magnetic field (i. e. its spatial intensity has an approximately Gaussian dependence in the axial direction and an integrated magnetic field is about $130 \mathrm{G} \cdot \mathrm{cm}$ ) which is strong enough to prevent all energetic primary electrons in the right chamber from entering the left chamber. Cold electrons, however, together with positive ions, can penetrate the filter and form a diffused plasma. Besides, a plasma grid whose potential is variable is added in the left chamber.

\section{Experimental results and discussion}

\subsection{Effect of the magnetic filter on controlling plasma parameters}

A remarkable change in plasma parameters (i. e. EEDF, electron density $n_{e}$, electron temperature $T_{e}$, plasma space potential $V_{s}$, floating potential $V_{f}$ etc.) across the magnetic filter has been clearly observed $^{(3) \sim(5)}$.

Fig. 2 shows a typical example of spatial variations of the EEDF, (a) the hydrogen plasma case and (b) the argon plasma case, respectively. The EEDF varies remarkably across the filter. In the diffused plasma region, there is little of the highenergy component of electrons. Namely, the magnetic filter prevents only the high-energy electrons from entering into the diffused plasma region. This feature is the most remarkable point on controlling the EEDF. In general, when the EEDF is controlled by using an electrostatic mesh grid, on the contrary, only slow electrons are reflected. Because, a retarding field is applied usually to control the EEDF.

Fig. 3 shows spatial variation of the high-energy component of electrons (i. e. the density ratio of fast to total plasma electrons $\left.n_{f e} / n_{e}\right)$, corresponding to the EEDF in Fig. 2 ( a ). From the EEDF, the density of fast electrons $n_{f e}(E)$ with an energy higher than $E$ was calculated. Corresponding to the variation of the EEDF, $n_{f e} / n_{e}$ in the diffused plasma decreases remarkably across the filter.

With the change of the filter position, those two distinct regions (i. e. the source plasma region and the diffused plasma region) are spatially well controlled $^{(3)(4)}$.

Even if $\mathrm{CH}_{4}$ gas is introduced into hydrogen or argon plasma, in both cases, the magnetic filter can spatially control well the EEDF and the related plasma parameters. Fig. 4 shows spatial variation 


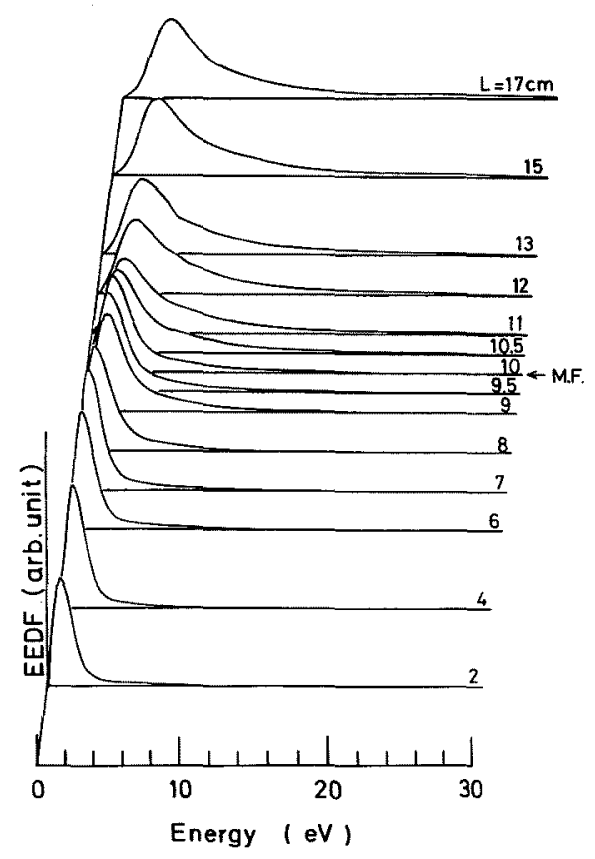

(a)

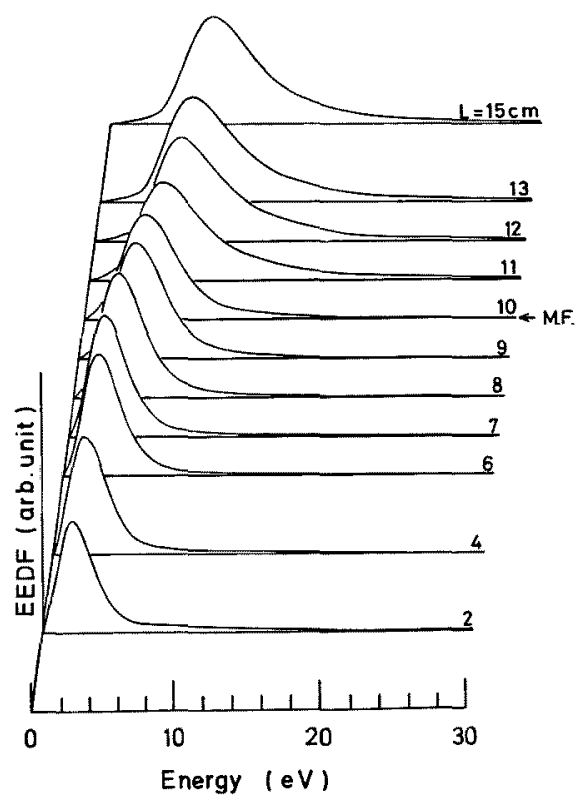

(b)

Fig. 2. Axial variations of the EEDF for two different cases, (a) hydrogen plasma and (b) argon plasma. Experimental conditions are as follows: discharge voltage $V_{d}=80 \mathrm{~V}$, discharge current $I_{d}=2 \mathrm{~A}^{\prime}, p\left(\mathrm{H}_{2}\right.$ or Ar gas $)=2.9 \mathrm{mTorr}$ and filter position $L_{f}=10 \mathrm{~cm}$.

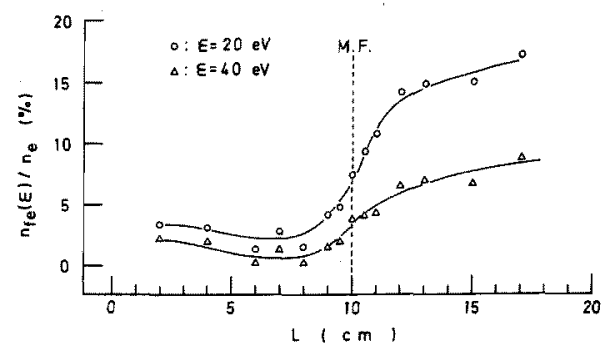

Fig. 3. Axial variations of density ratio $n_{f e} / n_{e}$ corresponding to the result shown in Fig. 2(a).

of $n_{e}$ in (a), $T_{e}$ in (b) and $V_{f}$ in (c). They vary across the filter. For example, there is a remarkable difference in $V_{f}$, and controlling $V_{f}$ is important in plasma processing. In plasma etching, $V_{f}$ is essential because it is directly related to the energy of the etching ions.

With increasing $p$, electron-neutral collisions may reduce the effect of the magnetic filter on controlling plasma parameters. The present two permanent magnetic filter rods produce an integ. rated magnetic field of about $130 \mathrm{G} \cdot \mathrm{cm}$. Up to $10 \mathrm{mT}$ orr, this magnetic field is strong enough to prevent primary electrons from passing(4), although optimum field strength depends on plasma conditions.

\subsection{Effect of the plasma grid on controlling plasma parameters}

We note that plasma parameters in the diffused plasma region strongly depend on the plasma grid potential $V_{b}^{(5)}$. With increasing $V_{b}(>0)$, as a whole, $n_{e}$ decreases monotonically, and $T_{e}, V_{s}$ and $V_{f}$ increase monotonically.

Fig. 5 shows a typical example of spatial variation of $n_{e}$ in (a) and $T_{e}$ in (b). Parameter is $V_{b}$. It is interesing that effect of changing $V_{b}$ appears not only in front of the plasma grid but also in the whole region of the diffused plasma.

From the experimental results presented in Sections 3.1 and 3.2, we suppose that plasma parameters are spatially well controlled by using both the movable magnetic filter and the potential varying plasma grid, and that this type of the plasma source is suitable for a tandem plasma processing 


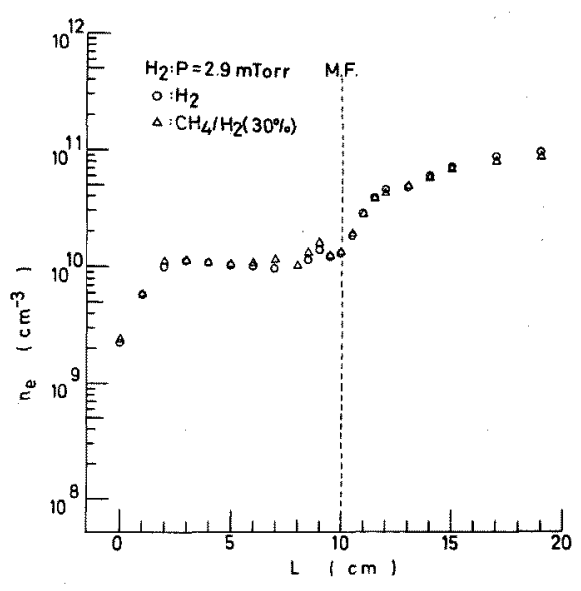

(a)

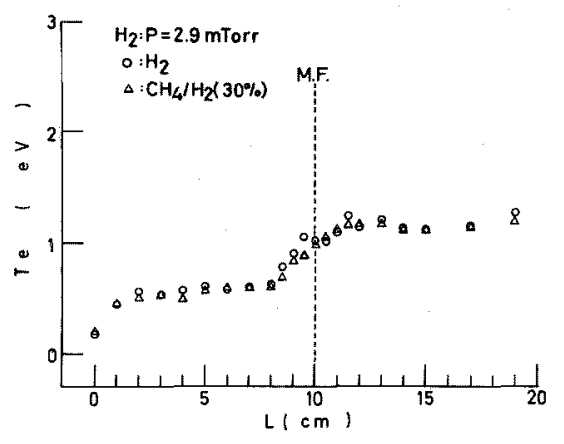

(b)

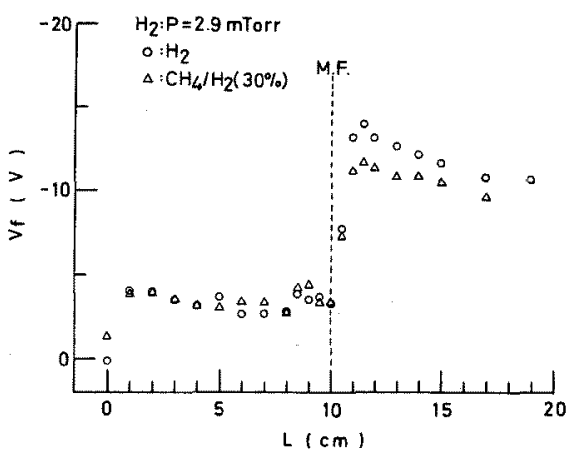

(c)

Discharge conditions are as follows: $V_{d}=80 \mathrm{~V}$ and $I_{d}=2 \mathrm{~A}$.

Fig. 4. Axial variations of some plasma parameters in $\mathrm{H}_{2}-\mathrm{CH}_{4}$ plasmas when $L_{f}=$ $10 \mathrm{~cm}$; (a) $n_{e}$, (b) $T_{e}$ and (c) $V_{f}$.

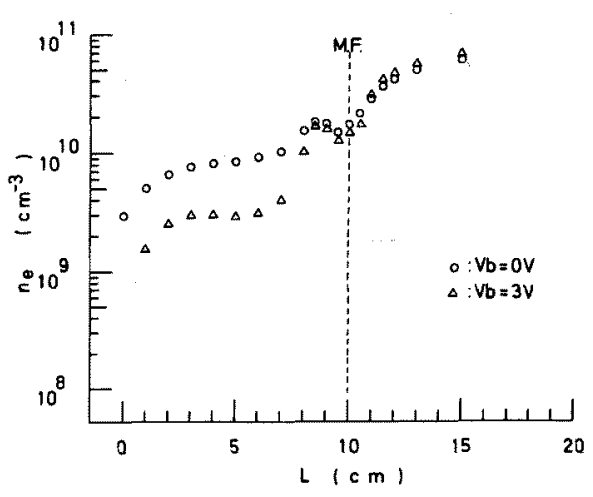

(a)

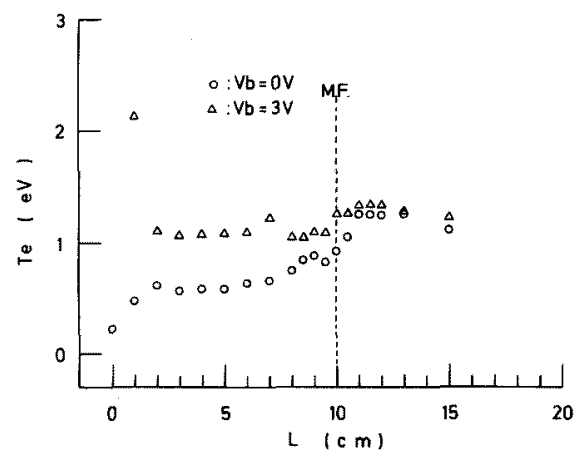

(b)

Experimental conditions are as follows: $V_{\alpha}=80 \mathrm{~V}, I_{\alpha}=$ 2A, $p\left(\mathrm{H}_{2} \mathrm{gas}\right)=2.9 \mathrm{~m}$ Torr and $L_{j}=10 \mathrm{~cm}$.

Fig. 5. Axial variations of $n_{e}$ in (a) and $T_{e}$ in (b) for two different plasma grid potentials $V_{b}$.

reactor.

\subsection{Particle simulation of magnetically filtered plasmas}

According to the experimental results reported in Section 3.1, the magnetic filter prevents only the high-energy electrons entering into the diffused plasma region. To clarify the role of the magnetic filter, we have undertaken a 2-dimensional particle simulation ${ }^{(6)}$. Characteristic features of preliminary results agree well with those of the experimental results.

Fig. 6 shows two examples of an electron motion, i. e. a trapped and an untrapped electrons. From these particle orbits, we could estimate diffusion 


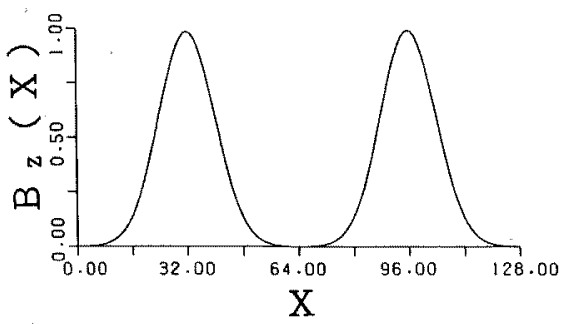

(a) The profile of the localized magnetic filter field $B_{z}(x)$.

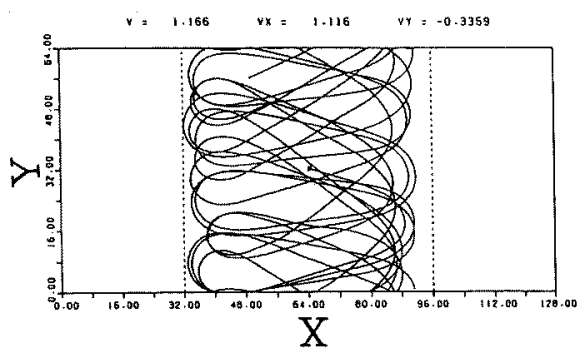

(b) An example of particle orbit for the trapped electron between two magnetic filters.

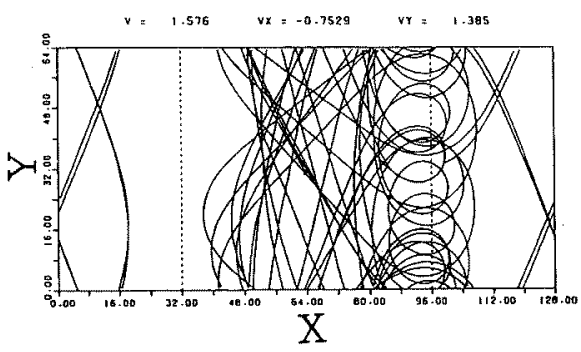

(c) An example of particle orbit for the untrapped electron.

Fig. 6. Particle simulation of magnetically, filtered plasmas in the two-dimentional system, i. e. the $X-Y$ plane.

coefficient $D(v)$ and the velocity distribution function of the penetrated electrons.

Fig. 7 shows $D_{x}(v)$ as a function of $v$, where $v^{2}=$ $v_{x}^{2}+v_{y}^{2} . D_{x}(v)$ is a decreasing function of an electron velocity $v$. Physical meaning is as follows: Most electrons cross the magnetic filter due to $\boldsymbol{E} \times \boldsymbol{B}$ drift, where $\boldsymbol{E}$ is the thermally excited fluctuation field and $\boldsymbol{B}$ is the filter field. Because of the finiteLarmor-radius effect, $\boldsymbol{E} \times \boldsymbol{B}$ drift for electrons decreases with the increase of $v$. Thus, $D_{x}(v)$ decreases with $v$, and energetic electrons can be reflected preferentially by the filter.

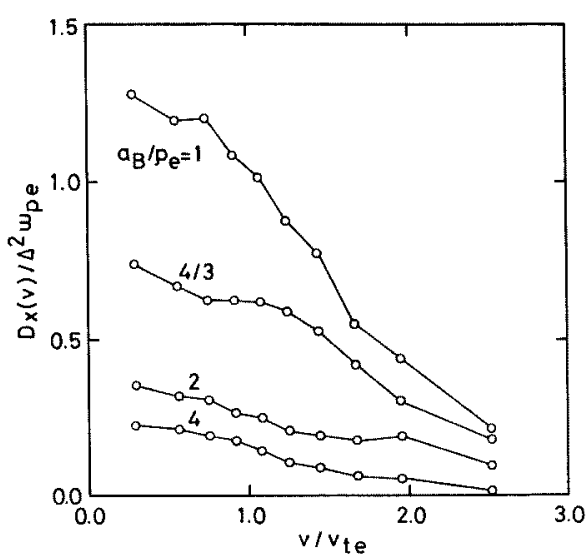

Parameter $a_{B} / \rho_{E}$ is the ratio of the characteristic width of the magnetic filter to the electron thermal Larmor radius for the maximum magnetic field.

Fig. 7. Velocity dependence of the diffusion coefficient $D_{x}(v)$ for the electrons.

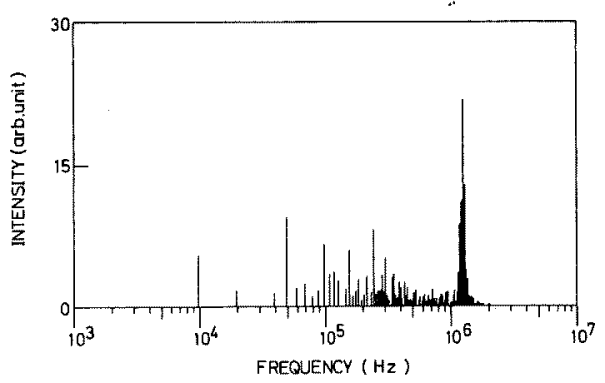

Experimental conditions are as follows: $V_{d}=80 \mathrm{~V}, I_{d}=$ $2 \mathrm{~A}, p\left(\mathrm{H}_{2}\right.$ gas $)=2.9 \mathrm{~m}$ Torr, $L_{f}=10 \mathrm{~cm}$, probe position $L=9.3 \mathrm{~cm}$ and probe bias potential $V_{p}=10 \mathrm{~V}$.

Fig. 8. A typical example of fluctuation frequency spectrum.

Fig. 8 shows a typical example of fluctuation spectrum, where probe position $L=9.3 \mathrm{~cm}$ and $L_{f}=$ $10 \mathrm{~cm}$. Plasma fluctuation with a well-defined frequency (i. e. 1-2 $\mathrm{MHz}$, a frequency range of lower hybrid waves) was observed when the probe was biased above the earth potential, and relative intensity in its amplitudes increases with increasing probe potential $V_{p}$. However, the ratio of electron density fluctuation $\Delta n_{e} / n_{e}$ is maximum at about plasma potential and decreases with increasing $V_{p}$. Therefore, we think that those fluctuations are excited spontaneously although they might have 


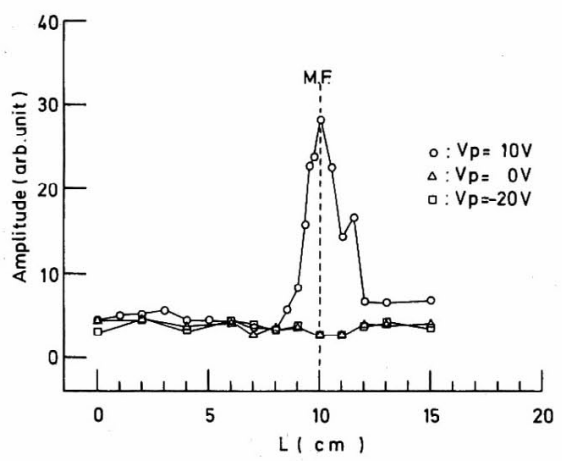

Experimental conditions are the same as those in Fig. 8.

Fig. 9. Axial variations of fluctuation amplitudes for three different probe bias potentials.

possibility to be excited by current-driven instability. Fig. 9 shows the axial dependence of fluctuation amplitudes. Apparently, fluctuation fields are localized in the vicinity of the magnetic filter. These results support the physical picture of the magnetic filter mentioned above, although details including identification of plasma fluctuation are now under study.

\section{Conclusions}

We have confirmed that the EEDF and other plasma parameters change steeply across the magnetic filter, and that the entire plasma is divided into to distinct regions (i. e. the source plasma with high-energy electrons and the diffused plasma without high-energy electrons). The former is suitable for production of the reactive species, and the latter is also suitable for deposition of thin films. In addition, we have confirmed that, by changing the plasma grid potential, the plasma parameters in the diffused plasma well controlled.

The role of the magnetic filter (i. e. preferential reflection of high-energy electrons) is studied by particle simulation, and we have obtained the qualitative agreement between the experimental and simulation results. Detailed discussion will be reported elsewhere.

In the future, we study further the relationship between the control of reactive plasmas (i. e. $\mathrm{CH}_{4}$ plasmas) and the formation of thin films, and the effect of negative ions on the kinetics in the reactive plasmas.

\section{Acknowledgements}

We would like to thank Professor R. Itatani for his continuing encouragement. We also thank $\mathrm{N}$, Shigeyama and H. Sakai for their assistance with the experiments. This work was partly supported by a Grant-in-Aid for Scientific Research on Control of Reactive Plasmas in Priority Areas, from the Ministry of Education, Science and Culture.

(Manuscript received May 2, '91)

\section{References}

(1) R. Itatani : J. IEE Japan (in Japanese), 110, 167(1990)

(2) R. Limpaecher \& K. R. Mackenzie: Rev. Sci. Instrum. 44 726 (1973)

( 3 ) O. Fukumasa: Proc. 6th Symp. Plasma Processing, Kyoto, p. 165 (1989)

(4) O. Fukumasa, H. Naitou \& S. Sakiyama: Japan. J. Appl. Phys. 30, L 1063 (1991)

(5) O. Fukumasa, N. Shigeyama, H. Sakai \& H. Naitou: Proc. 13th Symp. on Ion Sources \& Ion-Assisted Technol ogy, Tokyo, p. 97(1990)

(6) H. Naitou \& O. Fukumasa: Proc. 8th Symp. Plasma Processing, Nagoya, p. 133(1991)

(7) K. N. Leung, K. W. Ehlers \& M. Bacal : Rev. Sci. Instrum. 54, 56(1983)

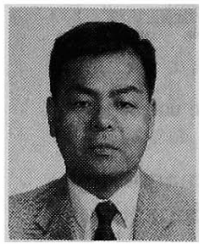

Osamu Fukumasa (Member)

He was born in Japan on January 8,1946 . He finished Doctor's Course, Division of Electrical Engineering, the Graduate'School of Engineering, Kyoto University in March 1973. He received Dr. Eng. from Kyoto University in 1981. During 1973 1982, he was Research Associate at Faculty of Engineering, Kyoto University. $\mathrm{He}$ became Lecturer in 1982, Associate Professor in 1983 and Professor in 1988 at Faculty of Engineering, Yamaguchi University. His research interests is in the field of plasma science, gas discharge physics and plasma chemistry.

Dr. Fukumasa is a member of Physical Society of Japan, the Japan Society of Plasma Science, Nuclear Fusion Research, and Illumination Engineering Society of Japan. 


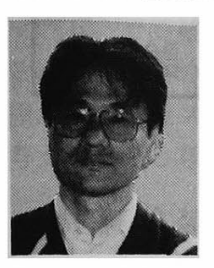

\section{Hiroshi Naitou (Member)}

He was born in Japan on March 9, 1952. He finished Doctor's Course, Division of Physics, the Graduate School of Science, Nagoya University in March 1979 . He received Dr. Sci. from Nagoya University in 1980. During 1979 $\sim 1989$, he was Research Associate at Institute of Plasma Physics, Nagoya University. He became Associate Professor in 1989 at Faculty of Engineering, Yamaguchi University. His research interests is in the field of plasma science, nuclear fusion and computer simulation.

Dr. Naitou is a member of Physical Society of
Japan, the Japan Society of Plasma Science and Nuclear Fusion Research.

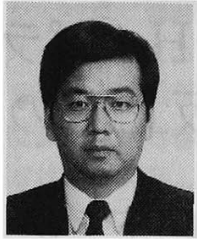

Satoshi Sakiyama (Member)

He was born in Japan on September 17, 1958. He finished Master's Course, Division of Electrical Engineering, the Graduate School of Engineering, Yamaguchi University in March 1983. He became Research Associate in 1983 at Faculty of Engineering, Yamaguchi University. His research interests is in the field of gas discharge physics and plasma chemistry.

He is a member of Physical Society of Japan. 